\title{
Thyrotoxicosis with ocular myasthenia - Case report
}

\author{
Dr. M Patel, Dr. N P Pothina
}

Scunthorpe General Hospital Scunthorpe DN15 7BH, UK

email: dr_meghna@icloud.com narayana.pothina@nhs.net•phone: 01724290104

\section{Introduction}

$>$ Graves's disease is characterised by a hyperfunctioning thyroid gland due to stimulation of the thyroid stimulating hormone receptor by autoantibodies directed against it. The other associated clinical manifestations include ophthalmopathy, dermopathy and acropachy.

$>$ Myasthenia gravis is a relatively rare autoimmune disorder in which antibodies form against the acetylcholine nicotinic post synaptic receptors at the neuro muscular junctions of skeletal muscles.

$>$ This can be ocular or generalised myasthenia. Association between these two disorders were reported in the past[1].

$>$ There is overlap of ocular clinical features in both these conditions which can pose a significant clinical challenge. The diagnosis is necessary for both therapeutic and prognostic reasons

\section{Case Report}

$>35$ year old lady presented to acute medical unit with diplopia on downward gaze for the last 4 weeks.

$>$ There is history of fatigue, unintentional weight loss of 13 kilograms over the last few weeks, variable mood and occasional palpitations.

$>$ On examination she is haemodynamically stable with mild tremor in both hands and no palpable goitre on neck examination.

$>$ She had vertical diplopia with mild right eye proptosis, lidlag and bilateral ptosis.

$>$ Systemic examination is unremarkable.

$>$ There is no evidence of pretibial myxoedema, proximal myopathy or acropachy.

\section{Investigations and Management}

Thyroid tests revealed hyperthyroidism with TSH <0.01 mU/L (0.27-4.2); Free T4 63.2pmol/L (12-22); Free T3 21.61 pmol/L (3.1 6.8);

$>$ TPO antibodies $117 \mathrm{IU} / \mathrm{ml}(0-34) \&$ Thyrotropin receptor antibodies $11.9 \mathrm{U} / \mathrm{L}(1-1.8)$ are elevated.

$>$ Her full blood count, renal function and liver function tests are within normal range.

$>$ Diagnosis of Graves Thyrotoxicosis is made and she was commenced with carbimazole, to be followed up in 4 weeks. At follow up there is good improvement in the thyroid function tests.

$>$ As the patient had ptosis with diplopia, MRI orbits were done which excluded the graves ophthalmopathy. Tensilon test and electromyography were done which confirmed ocular myasthenia.

$>\mathrm{CT}$ thorax did not reveal any thymoma. Acetylcholine Receptor Ab-1.3nmol/L(0-0.2) are elevated

$>$ She is treated with pyridostigmine with resolution of diplopia. She was referred to Neurology colleagues for further management of her myasthenia

\section{Discussion}

Autoimmune thyroid disease is present in $5-7.5 \%$ of myasthenia patients but myasthenia is present in only $0.2 \%$ of patients with thyrotoxicosis[2]. Graves disease is associated with ocular form of myasthenia gravis[3]. The reason for is association is unknown but several different hypothesis were postulated with a possibility of immunological cross reactivity \& genetic linkage[4]. Human leukocyte antigen(HLA) specificity B7, DR3 \& BW46 between myasthenia gravis and thyroid disease has been reported[5]. In 75\% of patients thyrotoxic symptoms appear much before or concurrently with the myasthenia symptoms[6]. The ocular manifestations in Graves disease include exophthalmos, periorbital oedema, lid lag, chemosis and ophthalmoplegia. Commonly involved extraocular muscles involved are superior and lateral recti[7]. Orbicularis oculi weakness in combination with ptosis or external ophthalmoparesis is prominent in myasthenia so the presence of ptosis is a good clinical clue for the possibility of myasthenia[8]. In this patient the ptosis is the giveaway sign which warranted further investigation to confirm the Myasthenia Gravis.

\section{Conclusion}

The main objective of this case is to highlight the importance of being clinically vigilant in thyrotoxic patient with ptosis to suspect underlying myasthenia and also to understand the association between Graves thyrotoxicosis and Myasthenia Gravis.

\section{References}

Ratanakorn, D. and A. Veijajiva, Long-term follow-up of myasthenia gravis patients with hyperthyroidism. Acta Neurologica Scandinavica, 2002. 106(2): p. 93-98.

Yaman, A. and H. Yaman, Ocular myasthenia gravis coincident with thyroid ophthalmopathy. Neurology India, 2003. 51(1): p. $100-101$.

Garlepp, M.J., et al., Autoimmunity in ocular and generalised myasthenia gravis. Journal of Neuroimmunology, 1981. 1(3): p. $325-332$.

Marinó, M., et al., Mild Clinical Expression of Myasthenia Gravis Associated with Autoimmune Thyroid Diseases. The Journal of Clinical Endocrinology \& Metabolism, 1997. 82(2): p. 438-443

WEETMAN, A.P. and A.M. McGREGOR, Autoimmune Thyroid Disease: Further Developments in Our Understanding. Endocrine Reviews, 1994. 15(6): p. 788-830.

Bartley, G.B., The epidemiologic characteristics and clinical course of ophthalmopathy associated with autoimmune thyroid disease in Olmsted County, Minnesota. Transactions of the American Ophthalmological Society, 1994. 92: p. 477-588.

7. Ali, A.S. and N.R. Akavaram, Neuromuscular disorders in thyrotoxicosis. American family physician, 1980. 22(3): p. 97-102.

8. Luchanok, U. and H.J. Kaminski, Ocular myasthenia: diagnostic and treatment recommendations and the evidence base. Current opinion in neurology, $2008.21(1)$ : p. 8-15. 